\title{
乌s \\ Student difficulties measuring distances in terms of wavelength: Lack of basic skills or failure to transfer?
}

\author{
Mila Kryjevskaia* \\ Department of Physics, North Dakota State University, Fargo, North Dakota 58105, USA \\ MacKenzie R. Stetzer ${ }^{\dagger}$ \\ Department of Physics and Astronomy \& Maine Center for Research in STEM Education, \\ University of Maine, Orono, Maine 04469, USA \\ Paula R. L. Heron \\ Department of Physics, University of Washington, Seattle, Washington 98195-1560, USA
}

(Received 27 October 2012; published 15 February 2013)

\begin{abstract}
In a previous paper that focused on the transmission of periodic waves at the boundary between two media, we documented difficulties with the basic concepts of wavelength, frequency, and propagation speed, and with the relationship $v=f \lambda$. In this paper, we report on student attempts to apply this relationship in problems involving two-source and thin-film interference. In both cases, interference arises from differences in the path lengths traveled by two waves. We found that some students (up to $40 \%$ on certain questions) had difficulty with a task that is fundamental to understanding these phenomena: expressing a physical distance, such as the separation between two sources, in terms of the wavelength of a periodic wave. We administered a series of questions to try to identify factors that influence student performance. We concluded that most incorrect responses stemmed from erroneous judgment about the type of reasoning required, not an inability to do said reasoning. A number of students do not seem to treat the spacing of moving wave fronts as analogous to immutable measurement tools (e.g., rulers).
\end{abstract}

DOI: 10.1103/PhysRevSTPER.9.010106

PACS numbers: 01.55.+b, 01.40.G-

\section{INTRODUCTION}

When two waves of the same amplitude and wavelength $(\lambda)$ arrive at a single point, the resulting disturbance depends on the phase difference between the waves $(\Delta \phi)$. In many situations, this phase difference arises from a difference in distances traveled by the waves $(\Delta D)$. Interferometry techniques - whether using acoustic, electromagnetic, matter, or gravitational waves-all exploit this phenomenon. The analysis of these experiments requires the application of the relationship among frequency, wavelength, and propagation speed ( $v=f \lambda$ in nondispersive media) and the relationship between phase difference and path length difference (e.g., $\Delta \phi=2 \pi \Delta D / \lambda$ for waves from two in-phase sources). Wosilait and collaborators in the University of Washington Physics Education Group (UW PEG) reported difficulties with the latter relationship in the context of the interference of water waves [1]. Recently we reported on

\footnotetext{
*mila.kryjevskaia@ndsu.edu

mackenzie.stetzer@maine.edu

pheron@phys.washington.edu
}

Published by the American Physical Society under the terms of the Creative Commons Attribution 3.0 License. Further distribution of this work must maintain attribution to the author(s) and the published article's title, journal citation, and DOI. difficulties that students experienced with the concepts of $\lambda, f$, and $v$ and their ability to reason with the relationship $v=f \lambda[2,3]$. Most of the evidence came from student responses to problems involving the transmission of mechanical waves from strings of one density to another, or from regions of one depth to another in a tank filled with water. We also reported that similar difficulties arise in the context of the interference of water waves from two sources, and in the context of thin-film interference. Apparently, additional exposure to the fundamental concepts in more advanced situations was not adequate to address the difficulties that emerged. In this paper we present additional results and show that some of the errors made by students in solving interference problems stem from difficulty with perhaps the most fundamental task: expressing a physical distance, such as the separation between two sources or slits, in terms of the wavelength of a periodic wave.

\section{INSTRUCTIONAL CONTEXT}

The investigation was carried out in introductory calculus-based courses at the University of Washington (UW) and at North Dakota State University (NDSU). The introductory courses at these two institutions differ in several ways, and tend to enroll students with somewhat 
different backgrounds. We provide data from both to illustrate that patterns in how students respond are not unique to either institution.

Most of the data were obtained from examination questions administered after instruction on the relevant concepts, including instruction based on Tutorials in Introductory Physics [4]. Other data were obtained from online pretests, which precede each tutorial but may (and often do) follow instruction in lecture and/or laboratory. Students receive credit for completing the pretests, whether or not their answers are correct (provided they demonstrate some effort). The online pretests typically require students to select an answer from a menu of options and then to type an explanation for their choice.

At UW the tutorials are used in small-group sessions led by graduate teaching assistants, as has been described elsewhere. (For example, see [5,6].) At NDSU, there are not enough teaching assistants available to support small-group discussion sessions. Instead the tutorials are used as a basis for interactive lectures. (For discussion of the use of tutorials as the basis for interactive lectures in large classes, see $[7,8]$.) Modified tutorial worksheet questions are presented one by one on a screen and all students consider a specific question simultaneously. An instructor poses a question and students volunteer their answers. Questions critical for the development of ideas are presented in a "peer instruction" format: multiple-choice answers are displayed on the screen, students discuss their reasoning with each other, and choose their individual answers using hand-held personal response devices, or "clickers" [9]. After the results of the polling are revealed, students are encouraged to support their answers in a whole-class discussion.

In the sequence of tutorials that deals with waves, interference is introduced in the context of water waves. In the tutorial Two-source interference, students become familiar both with the diagrammatical representation of circular wave fronts and with the task of determining a distance (such as the source separation $d$ ) in terms of the wavelength $\lambda$. Students practice measuring distances and differences in distances in terms of $\lambda$ in the case of twosource interference of water waves and articulate conditions necessary to observe nodal lines and lines of maximum constructive interference. Students also discuss how an interference pattern depends on (1) the source separation $d$ (e.g., as $d$ measured in terms of $\lambda$ increases, the number of interference lines increases as well) and (2) the frequency of the two (in-phase) sources (e.g., an increase in $f$ results in decrease in $\lambda$ which leads to a greater $d$ in terms of $\lambda$ ).

In the tutorial Wave properties of light, students extend their understanding of interference of water waves to the more abstract context of light. From an observation of a periodic plane water wave incident on a barrier with a small opening of size $a$ students conclude that as $a$ decreases, the transmitted waterfronts become "more circular." At a certain limit, an opening could be treated as a point source of circular water waves. Students recognize that ideas developed in the context of two-source interference are directly applicable to the context of waves incident on a mask with two very narrow slits. Students then make an explicit connection between two-slit interference of water waves and light.

The tutorial Thin-film interference builds upon ideas developed in prior tutorials on wave behavior at the boundary and interference. In the tutorial, students are presented with an opportunity to integrate their understanding of these concepts. The tutorial is not designed to target specific student difficulties with either wave behavior at a boundary or interference phenomena. Rather, it guides students through the reasoning necessary to recognize how thin-film interference occurs.

\section{PROBLEMS INVOLVING TWO-SOURCE AND THIN-FILM INTERFERENCE}

As part of the development and assessment of the sequence of tutorials on waves and physical optics, we have designed a variety of examination problems. Here we give two examples in which students were asked to predict how an interference pattern changes, if at all, after certain specified changes are made to an experimental setup. One example involves water waves; the other, thin-film interference.

\section{A. Analyzing changes to a two-source interference pattern}

In the two-source interference problem, students were shown a diagram depicting periodic waves generated by two point sources that are in phase in a large tank of water [see Fig. 1(a)]. The problem included five questions; the first three $(\mathrm{A}-\mathrm{C})$ deal with the situation shown in the diagram. Students were first asked to determine the distance between the sources $d$ in terms of the wavelength $\lambda$. Next they were asked to determine the number of nodal lines and lines of maximum constructive interference shown in Fig. 1(b). Then they were asked to calculate the angle $\theta$ (relative to a normal that bisects the two sources) at which the first nodal line occurs in the region far away from the sources. To answer the first part correctly, students could simply count the intervals between adjacent crests and troughs (indicated by solid and dashed lines) that occur between the two sources to determine the sources are $2 \lambda$ apart. Given this separation (and the fact that the sources are in phase), they could reason that the maximum possible difference in distances to the two sources $\left(\Delta D_{\max }\right)$, which occurs for points along the line that contains the two sources, is $2 \lambda$, and that this is therefore a line of maximum constructive interference. It follows that there are also lines of maximum constructive interference corresponding to path length differences $\Delta D=0$ and $\lambda$, and nodal lines corresponding to $\Delta D=\lambda / 2$ and $3 \lambda / 2$. The relationship 
(a)

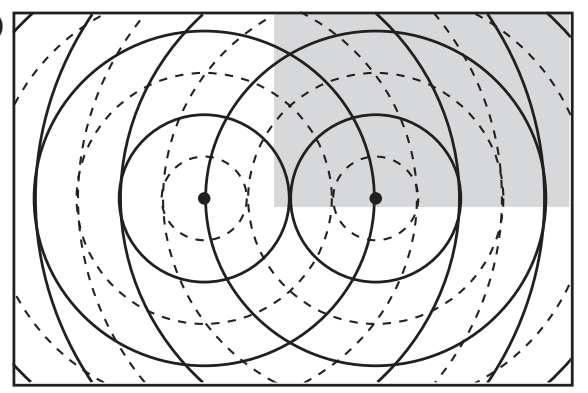

(b)

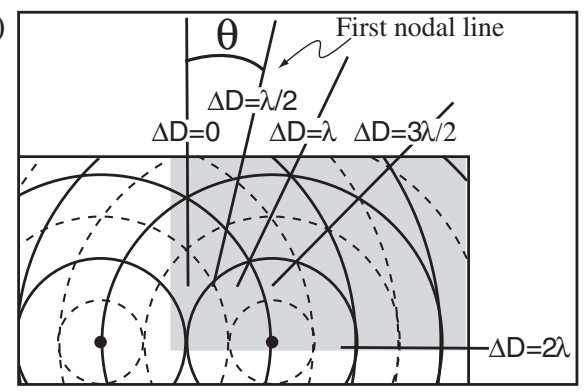

FIG. 1. (a) Diagram shown to students in the two-source interference problem. As part of the problem, students determine the number of nodal lines and lines of maximum constructive interference in the shaded region and the angle $\theta$ at which the first nodal line occurs in the region far away from the sources. (b) Diagram showing the features to be identified by students.

$d \sin \theta=\Delta D$ along with $d=2 \lambda$ and $\Delta D=\lambda / 2$ can then be used to determine that $\theta \simeq 14.5^{\circ}$ for the first nodal line.

The final two questions ( $\mathrm{D}$ and $\mathrm{E}$ ) deal with changes made to the original situation. In case 1, the propagation speed of the waves in the tank is decreased by a factor of 2 $\left(v_{\text {modified }}=v_{\text {original }} / 2\right)$; in case 2 , the rate at which the sources tap the surface of the water is halved $\left(f_{\text {modified }}=\right.$ $f_{\text {original }} / 2$ ). In both cases no other changes are made to the experimental setup. For each case, students were asked to determine whether the first nodal line of the original pattern will become a line of maximum constructive interference, remain a nodal line, or be neither.

For both cases, students had to recognize that in the original experiment, the difference in the distances traveled by the waves from the two sources to points on the first nodal line $\Delta D$ is equal to $\lambda_{\text {original }} / 2$. In case $1, v$ is decreased by a factor of 2 , while $f$ is kept constant, so $\lambda_{\text {modified }}=\lambda_{\text {original }} / 2$ and thus $\Delta D=\lambda_{\text {modified }}$. Therefore, after the change, waves arriving at points corresponding to the original first nodal line are in phase, and thus these points form a line of maximum constructive interference. In case 2 , after $f$ is decreased by a factor of 2 , while $v$ is kept constant, $\lambda_{\text {modified }}=2 \lambda_{\text {original }}$, and $\Delta D=\lambda_{\text {modified }} / 4$. As a result, the path length difference for waves arriving at points corresponding to the original first nodal line is neither zero nor an integer multiple of $\lambda / 2$. Therefore these points no longer constitute a nodal line nor a line of maximum constructive interference.

These questions were given on a midterm exam at UW after students had completed the tutorials Two-source interference, Wave properties of light, and Multiple-slit interference, as well as instruction on interference in lecture (with accompanying homework assignments) and in laboratory. At NDSU, these questions were given as part of the final exam after laboratory and interactive lectures based on this set of tutorials (with accompanying homework assignments) [10].

Almost all of the students ( $>90 \%$ ) correctly determined the distance between the sources (see Table I). Most students $(68 \%$ at UW and $61 \%$ at NDSU) correctly determined the number of nodal lines and lines of maximum constructive interference. About half of the UW students correctly calculated the angle to the first nodal line [11]. NDSU students performed significantly worse on this part of the question: only $18 \%$ responded correctly with correct reasoning.

At both universities, students were least successful on the final two questions (D and E). (See Table II.) Few students (22\% at UW and $20 \%$ at NDSU) provided qualitatively correct reasoning on one or both tasks that involved recognizing that a change in $\lambda$ leads to changes in relevant distances measured in terms of $\lambda_{\text {modified }}$. Students at UW

TABLE I. Summary of results from questions A-C of the two-source interference problem after all instruction on two-source interference.

\begin{tabular}{lcc}
\hline \hline & $\begin{array}{c}\text { UW } \\
N=153\end{array}$ & $\begin{array}{c}\text { NDSU } \\
N=90\end{array}$ \\
\hline $\begin{array}{l}\text { Question A: Distance between the sources in terms of } \\
\text { Correct answer }(d=2 \lambda) \text { with correct reasoning }\end{array}$ & $94 \%$ \\
Question B: The number of nodal lines and the lines of maximum constructive interference \\
in the shaded area far away from the sources \\
$\quad$ Correct answer with correct reasoning \\
$\begin{array}{l}\text { Question C: The angle to the first nodal line in the far away region } \\
\quad \text { Correct answer with correct reasoning }\end{array}$ \\
\hline \hline
\end{tabular}


TABLE II. Summary of results from questions D and E of the two-source interference problem after all instruction on two-source interference. Indented categories are subcategories of the preceding major category.

\begin{tabular}{|c|c|c|}
\hline & $\begin{array}{c}\mathrm{UW} \\
N=153\end{array}$ & $\begin{array}{l}\text { NDSU } \\
N=90\end{array}$ \\
\hline Qualitatively correct reasoning on at least one question & $22 \%$ & $20 \%$ \\
\hline Correct answer with correct and complete reasoning on both questions & $22 \%$ & $6 \%$ \\
\hline $\begin{array}{l}\text { Correct answer on both questions with incomplete reasoning on question D: } \\
\text { " } d \text { in terms of } \lambda \text { increases, the \# of lines increases" }\end{array}$ & None & $14 \%$ \\
\hline \multicolumn{3}{|l|}{ Types of incorrect reasoning (on one or both questions) } \\
\hline Incorrect relationship among $v, f$, and $\lambda$ & $23 \%$ & $26 \%$ \\
\hline Difficulties with the concept of interference & $27 \%$ & $13 \%$ \\
\hline Incorrect $d$ or $\Delta D$ in terms of $\lambda$ & $9 \%$ & $8 \%$ \\
\hline
\end{tabular}

appropriately focused on determining the effect of the change in $\lambda$ on the path length difference and therefore provided correct and complete arguments in both cases. At NDSU, however, students focused on the distance between the sources. This reasoning approach leads to a correct and complete argument for case 2 only $\left(f_{\text {modified }}=f_{\text {original }} / 2\right)$. In this case, since $\lambda_{\text {modified }}=2 \lambda_{\text {original }}, d$ in terms of $\lambda$ decreases $\left(d=\lambda_{\text {modified }}\right)$; as a result, the first nodal line moves away from the central maximum such that the region of the original nodal line now lies between the central maximum and the first nodal line. Similar reasoning applied to case $1\left(v_{\text {modified }}=v_{\text {original }} / 2\right)$ leads to an incomplete argument. Many students assumed that since the number of lines in the tank increases ( $d$ changes from $2 \lambda_{\text {original }}$ to $\left.4 \lambda_{\text {modified }}\right)$ resulting in the first nodal line moving closer to the central maximum, the first maximum must take its place.

\section{Nature of student errors}

On the final two questions, approximately $25 \%$ of the students failed to apply the relationship $v=f \lambda$ correctly. (See Table II.) As we reported previously [3], some students incorrectly predicted that the change in propagation speed (case 1) affects both wavelength and frequency, or that the change in frequency (case 2) affects propagation speed.

A significant fraction of the students incorrectly applied the model for two-source interference. However, some of these responses may also reflect a lack of understanding of the relationship $v=f \lambda$. Some students claimed that, since the same change occurs to both sources (or waves), the interference pattern is not affected. For example, one student explained that after the propagation speed is decreased by a factor of 2 , the nodal line in the original experiment remains a nodal line because:

Both waves from two sources decrease [in] speed. So the resulting picture looks like the original, hence, nothing changes.

It appears that this student is referring to the diagram depicting circular waves rather than focusing on the interference pattern itself. The student failed to recognize that, as a result of the decrease in the propagation speed by a factor of 2, the wavelength of both waves also decreases by a factor of 2, so that the resulting "snapshot" of the interfering waves looks different: every line on the diagram that used to represent a trough must now represent a crest. Consequently, every nodal line in the original interference pattern now becomes a line of maximum constructive interference. The student quoted above, and others who gave similar responses, might have imagined a diagram that is a "scaled down" version of the original. However, the physical distance between the sources is not changed and therefore cannot be "scaled." Some students applied reasoning along these lines to one of the two cases while using a correct approach in the other. It may be that they failed to recognize that both propagation speed and frequency determine the wavelength of the waves.

Another category of incorrect responses, given by about $10 \%$ of the students, is characterized by the use of incorrect expressions for the relevant distances (either $d$ or $\Delta D$ ) in terms of $\lambda_{\text {modified }}$. (See Table II.) For example, one student explained that, after the propagation speed is decreased by a factor of 2, the first nodal line in the original experiment is neither a nodal line nor a line of maximum constructive interference because:

Changing the velocity decreases the wavelength by a factor of 2. This changes the source separation to $\lambda$, causing nothing to happen there. $\lambda / 2=\lambda \sin \theta$, $\theta=30^{\circ}$.

The only error in this response concerns the determination of the distance between the sources $d$ in terms of $\lambda_{\text {modified }}$. Apparently some students used their initially correct relationship $d=2 \lambda$ (where $\lambda$ is the original wavelength) and then "plugged in" $\lambda / 2$ (which corresponds to $\lambda_{\text {modified }}$ expressed in terms of $\lambda_{\text {original }}$ ) to obtain $d=\lambda$, implying that the distance between the sources has, in absolute terms, decreased [12].

The results from this problem indicate that student difficulties with the relationship among $v=f \lambda$ are persistent and emerge in a variety of different contexts. While we had anticipated some of the difficulties we observed, we did not 


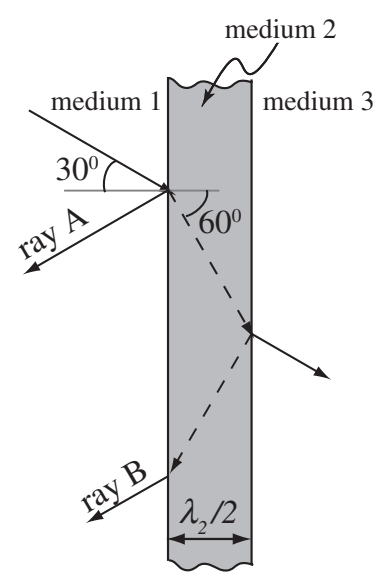

FIG. 2. Diagram shown to students as part of the thin-film interference problem.

expect that measuring a distance in terms of wavelength would be challenging for students. The results from the first part of the problem, in which more than $90 \%$ of the students correctly expressed the source separation in terms of $\lambda$, seemed to confirm this expectation. However, the occurrence of difficulties in later parts of the problem prompted us to examine responses from related problems that involved different physical situations.

\section{B. Analyzing changes to a thin-film interference experiment}

Understanding thin-film interference requires students to apply concepts associated with wave behavior at a boundary and to relate distances traveled in a medium to the wavelength in that medium. On the thin-film interference problem, students were given the ray diagram shown in Fig. 2. They were asked to consider a periodic wave passing from medium 1 through medium 2 into medium 3. A sequence of questions asked them to analyze various aspects of wave reflection, refraction, and interference. We limit the discussion here to a question specifically designed to probe student ability to apply the relationship among $v=f \lambda$ and to measure distances traveled by waves in a medium in terms of $\lambda$. On this question, students were told to consider a new experiment in which medium 1 is replaced with a different medium such that the wavelength of the incident wave is doubled while neither the angle of incidence on the left boundary nor the source of light is changed. They were asked to determine whether the distance traveled by the wave in medium 2 , measured in units of the wavelength in that medium, would increase, decrease, or remain the same [15].

To answer correctly, students needed to recognize (1) that the doubling of the incident wavelength must have been the result of a change in the propagation speed of the incident wave (i.e., medium 1 is modified) and (2) that the frequency of the incident light remained the same (because the source was unchanged). They could then reason that the angle of refraction in medium 2 must decrease and that, as a result, the distance traveled by the wave in medium 2 in absolute terms must also decrease. The wavelength in medium 2 is unaffected by the change to medium 1 so the distance traveled in medium 2 in terms of $\lambda_{2}$ is also decreased.

The question was given to UW students on a final exam upon completion of all course instruction, including instruction on thin-film interference in lecture and tutorial.

As outlined above, a correct solution to this problem requires several steps. Therefore it is perhaps not surprising that only $4 \%$ of the students answered correctly with correct reasoning. However, the errors made are revealing. Here we discuss a few that occurred frequently.

\section{Nature of student errors}

As reported previously [3,16], approximately $25 \%$ of the students failed to apply the relationship $v=f \lambda$ correctly. Most of these students apparently assumed that the transmitted wavelength must change if the incident wavelength has changed.

Many students (30\%) gave partially correct responses in which there is explicit mention of the change in physical distance only. (See Table III.) These students indicated that, in the new experiment, the distance traveled inside medium 2 changes as a result of the change in the angle of refraction $\left(\theta_{r \text { medium } 2}\right)$. However, there was no evidence of an attempt to express this distance in units of $\lambda_{2}$. Most of these students argued that "The light will be refracted at angle $\theta_{2}$, which is smaller, so the path in medium 2 will

TABLE III. Summary of results from the thin-film interference problem after all instruction at UW.

\begin{tabular}{lc}
\hline \hline & UW \\
& $N=124$ \\
\hline The distance in medium 2 in terms of $\lambda_{2}$ decreases because $\theta_{r}$ decreases & $4 \%$ \\
and $\lambda_{2}$ is unchanged. (Correct answer with correct reasoning.) & $27 \%$ \\
Incorrect application of $v=f \lambda: \lambda_{2}$ changes & $30 \%$ \\
Only considered how physical distance is affected by the change & $16 \%$ \\
Only considered how wavelength in medium 2 is affected by the change & $24 \%$ \\
Considered changes in both $d$ and $\lambda$ but made various errors &
\end{tabular}


decrease." It appears that they either did not know how to express the distance in terms of $\lambda$ or did not see the difference between being asked how the distance changes and how the distance measured in units of $\lambda$ changes.

A further $16 \%$ of the students considered the transmitted wavelength only. Responses in this category contained no mention of changes in the physical distance traveled by the wave in medium 2 . Many simply argued that the distance in terms of $\lambda_{2}$ remains the same because $\lambda_{2}$ remains the same in both experiments. Although the latter is correct, these students did not take into account that the physical distance traveled by the transmitted wave changes.

Only $28 \%$ of all students attempted to consider the effect of the change in the incident wavelength on both the distance traveled in medium 2 and the transmitted wavelength in order to express $d$ in terms of $\lambda_{2}$; the responses of most of the others did not indicate whether they had even attempted to do so. As stated above, of those who tried, only $4 \%$ succeeded while the rest made errors $(24 \%)$.

\section{PROBLEMS SPECIFICALLY DESIGNED TO PROBE STUDENT ABILITY TO RELATE DISTANCE TO WAVELENGTH}

The results above indicate that calculating distances in terms of wavelength is difficult for some students. In this section we present results from a series of questions that we designed in an effort to better understand the nature of such difficulties as well as the circumstances in which students would encounter these difficulties. We noted that errors that reflected incorrect measurement of distance emerged most clearly when students were required to consider a change to the original situation. Suspecting that the additional steps in reasoning could be a factor, we designed tasks that simply ask students to measure a distance. Below we present a few illustrative examples.

In order to understand the influence of instruction on student responses, we administered one of the problems (the "slit separation" task) to groups of students who were at different stages of instruction. In order to understand the influence of features of the questions themselves, we also administered a set of related problems to groups of students who were at the same stage of instruction. The groups of students were all enrolled in different sections of the same course, therefore no students had seen the same question more than once. Many previous investigations indicate that comparisons between different classes are valid, provided that caution is employed when differences are small [17].

\section{A. Measuring the distance between two slits in a barrier}

As shown in Table I, the first part of the two-source interference task, which uses a context and representation directly from the tutorial Two-source interference, did not present a challenge to students. Therefore, we set one of the new tasks in a different context: parallel wave fronts incident on two slits.

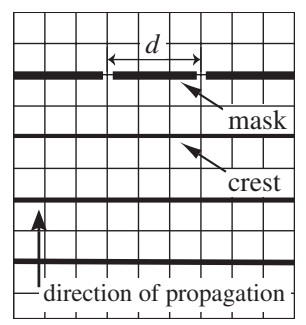

FIG. 3. To-scale diagram shown to students on the slit separation task.

The slit separation task focused on a periodic wave generated by a dowel in a tank of water. The students were shown the top-view diagram presented in Fig. 3, which depicts parallel wave fronts incident on a mask with two narrow slits. (The context of periodic water waves generated by a dowel was familiar to the students from the sequence of tutorials on mechanical waves.) They were asked to determine the slit separation in terms of the wavelength. As in the case of the first part of the twosource interference problem, the answer, $d=1.5 \lambda$, can be found by inspecting the figure.

The question was originally given as a part of a midterm exam at UW upon completion of two relevant tutorials: Two-source interference and Wave properties of light. At NDSU, this question was included on the final exam after interactive lectures based on these two tutorials. None of the students had seen the two-source interference problem discussed above although they had practiced similar reasoning during the tutorials.

At both institutions, about $60 \%$ of the students answered correctly and supported their answers with correct reasoning. (See Table IV.) The most common incorrect approach (used by $31 \%$ of the UW students and $16 \%$ of the NDSU students) involved mathematical expressions such as $d=m \lambda / \sin \theta$ or $d=m \lambda L / y_{m}$ (the latter is a formula presented in lecture for determining the location of nodes in an interference pattern on a distant screen). Some of the students who wrote such equations explained

TABLE IV. Summary of results from the slit separation task after all instruction on two-source interference.

\begin{tabular}{lcc}
\hline \hline & UW & NDSU \\
& $N=484$ & $N=161$ \\
\hline Correct answer $(d=1.5 \lambda)$ & $58 \%$ & $61 \%$ \\
With correct reasoning & $58 \%$ & $61 \%$ \\
With incorrect reasoning & None & None \\
Incorrect answer & $40 \%$ & $40 \%$ \\
With correct approach & $\sim 0 \%$ & $<5 \%$ \\
With various mathematical expressions & $31 \%$ & $16 \%$ \\
With incorrect $d$ in terms of $\lambda$ & $4 \%$ & $10 \%$ \\
(such as $d=\lambda$ ) & & \\
"Not enough information" & $\sim 0 \%$ & None \\
Other or blank & $5 \%$ & $12 \%$ \\
Total with correct approach & $59 \%$ & $61 \%$ \\
\hline \hline
\end{tabular}




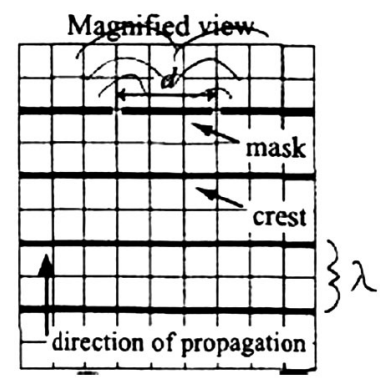

FIG. 4. A sketch given by a student in response to the slit separation task. The student correctly identified the wavelength on the diagram and yet failed to determine the distance between the slits in terms of the wavelength.

what each variable is and how it could be determined. Moreover, some who approached the problem from a mathematical perspective explicitly identified the distance between two consecutive crests as the wavelength, as shown in Fig. 4, but still failed to complete the basic task of measuring the distance $d$ in terms of $\lambda$.

The discrepancy between the results of the slit separation task ( $\sim 60 \%$ correct with correct reasoning) and those obtained on the first part of the two-source interference task ( $>90 \%$ correct with correct reasoning) is striking. (See Tables I and IV.) Below we probe several possible reasons for this discrepancy.

\section{Role of instruction that emphasizes a mathematical approach to interference}

We recognize that the relatively poor performance on the slit separation task might be due to students' tendency to rely on math (as in the traditional approach typically used to describe two-slit interference) rather than on conceptual reasoning (stressed in tutorials). (For example, see $[3,18,19]$.) Specifically, they may "frame" the first part of the two-source interference problem as one requiring the conceptual approach that had been emphasized in tutorial, but frame the slit separation problem as one requiring a mathematical approach. Thus, the errors made on the latter task may not necessarily signify a lack of basic understanding of the concept of wavelength or a lack of ability to express $d$ in terms of $\lambda$. Instead it may reflect the context-sensitive activation of epistemological resources based on students' expectations of the kinds of skills and knowledge necessary to complete a specific task (e.g., two-slit interference problems require the use of equations) and the associated conceptual resources (e.g., the identification of an equation that relates $d$ and $\lambda$ ) [20]. We reasoned that if this were the case, performance might be better prior to the introduction of a mathematical approach that may have informed student expectations. Thus, we administered the slit separation task on a webbased "pretest" for the tutorial Wave properties of light at UW [21]. The task therefore (1) followed the tutorial Two-source interference, in which students practiced applying conceptual reasoning based on the measurement of distances in terms of $\lambda$, but (2) preceded the introduction of mathematical formalism in the subsequent homework as well as in lecture and laboratory instruction. Students were given a selection of values for $d$ in terms of $\lambda$ as well as the opportunity to type any answer, such as a mathematical expression, for their response. They were also asked to explain their reasoning.

Only $49 \%$ of UW students answered correctly, most of whom (41\% of the total) gave correct explanations. (See column After-Before in Table V.) An additional 8\% of the responses showed evidence of a correct approach but a failure to examine the diagram carefully, as illustrated by the following response: " $d=\lambda$. One wavelength is about two squares and that is how far the slits are separated." In this case, the student carelessly estimated the distance between the slits as being equal to 2 squares as opposed to 3 squares.

TABLE V. Summary of results from the slit separation task at different stages of instruction at UW and NDSU. (Some of these data also appear in other tables; they are repeated here to facilitate comparisons.)

\begin{tabular}{|c|c|c|c|c|}
\hline \multirow[t]{3}{*}{$\begin{array}{l}\text { Timing relative to tutorial instruction (graphical or conceptual approach) } \\
\text { Timing relative to mathematical analysis of interference in lecture, lab, or tutorial }\end{array}$} & \multirow{2}{*}{$\begin{array}{l}\text { Before } \\
\text { Before }\end{array}$} & \multirow{2}{*}{$\begin{array}{c}\text { After } \\
\text { Before }\end{array}$} & \multicolumn{2}{|c|}{$\begin{array}{l}\text { After } \\
\text { After }\end{array}$} \\
\hline & & & UW & NDSU \\
\hline & $N=315^{\mathrm{UW}} \quad N=158$ & $N=158$ & $N=484$ & $N=161$ \\
\hline Correct answer $(d=1.5 \lambda)$ & $49 \%$ & $49 \%$ & $58 \%$ & $61 \%$ \\
\hline With correct reasoning & $35 \%$ & $41 \%$ & $58 \%$ & $61 \%$ \\
\hline With incorrect reasoning & $14 \%$ & $8 \%$ & None & None \\
\hline Incorrect answer & $51 \%$ & $51 \%$ & $42 \%$ & $39 \%$ \\
\hline With correct approach & $3 \%$ & $8 \%$ & $1 \%$ & $\sim 0 \%$ \\
\hline With various mathematical expressions (e.g., $d=m \lambda / \sin \theta$ ) & $0 \%$ & $<5 \%$ & $31 \%$ & $16 \%$ \\
\hline With incorrect $d$ in terms of $\lambda$ & $39 \%$ & $26 \%$ & $4 \%$ & $10 \%$ \\
\hline "Not enough information" & $9 \%$ & $10 \%$ & $\sim 0 \%$ & None \\
\hline Other or blank & $\sim 0 \%$ & $\sim 0 \%$ & $5 \%$ & $12 \%$ \\
\hline Total with correct approach & $\mathbf{3 8} \%$ & $49 \%$ & $\mathbf{5 9} \%$ & $61 \%$ \\
\hline
\end{tabular}


The overall success rate $(41 \%$ correct with correct reasoning) was lower than when the problem was given after additional instruction (58\% correct with correct reasoning as shown in column After-After in Table V), although the gap narrows when the percentage who attempted a correct approach is considered ( $49 \%$ on the earlier version versus a bit more than 59\% with more instruction). A comparison of overall success rates therefore does not support the notion that additional instruction emphasizing mathematics actually has a deleterious effect on student performance [22]. However, an examination of the incorrect answers does suggest that this instruction affects how students respond.

Responses that included mathematical expressions such as $d=m \lambda / \sin \theta$ were almost completely absent on the version given prior to the introduction of a mathematical approach (column After-Before in Table V), but represented $31 \%$ of the responses on the version given later (column After-After for UW data). Thus the results from the slit separation task given at different points during instruction support the idea that the introduction of mathematical analysis has an impact, but it appears that the effect is to provide students with an alternative incorrect approach (one that they may view as more sophisticated), rather than distracting them from applying a commonsense approach.

\section{Role of instruction on interference more generally}

The version of the slit separation task given at the earlier stage explicitly offered the students the option "there is not enough information to answer" (10\% of the students chose this response as shown in Table V). Some explained:

Those slits could be any distance apart and are not dependent on the wave, they are part of the system you have provided.

It appears that some students assume that only variables that depend on one another can be expressed in terms of each other.

Other students argued that they did not know enough about the interference pattern that would result when the waves emerge from the slits:

[More information is needed because I] don't know the interference pattern after the waves hit the slits.

The water wave didn't even cross the two slits yet, so there's not enough information to determine " $d$."

These students may be recalling the tutorial Two-source interference, in which they practiced determining the distance between sources in terms of the wavelength in situations in which the shapes of the interfering (circular) wave fronts were known. Without having any information about either the interference pattern or the shapes of the interfering wave fronts, perhaps they did not see how to perform this very basic task.

These responses suggested the possibility that after instruction on interference, regardless of its emphasis, some students tend to frame any interference-related tasks as being directly related to the tutorial. As such, it may lead to an activation and retrieval of those conceptual resources directly related to recent tutorial instruction instead of a more commonsense approach to measuring distance. The slit separation task, although it does not contain any references to interference, may, by its very nature, tend to invoke thinking in those terms. We reasoned that if this were the case, students might be more likely to answer the task correctly prior to any instruction on interference, but after the concept of wavelength had been covered. Therefore, we have also given the task prior to both the Two-source interference tutorial and lecture instruction on interference between waves from two sources at UW [23]. (Standing waves on strings had been discussed, but the interference of sound waves, water waves, or light waves had not yet been introduced.)

As shown in column Before-Before of Table V, 35\% of the students answered correctly with correct reasoning prior to instruction. Essentially the same success rate was obtained after some instruction on interference $(41 \%$, as shown in column After-Before). Thus, no positive shift in student performance was observed when the task was administered prior to instruction on interference. The above results do not support the idea that additional instruction on the topic leads students to assume that the task requires the application of concepts related to interference, and thereby diverts them from measuring distances using more basic approaches.

\section{Role of the physical situation: Different examples of interference}

As we have noted, there was a significant discrepancy between student performance on the first part of the twosource interference problem $(>90 \%$ correct with correct reasoning) and the slit separation task ( $\sim 60 \%$ correct with correct reasoning) given at similar stages in instruction. (See Tables I and IV.) Above we discussed two possible explanations, both based on the notion that exposure to certain types of instruction might lead students to attempt incorrect approaches. Neither explanation satisfactorily explained the results. We also note that the success rate on the slit separation task at UW is essentially the same before and after the two-source interference tutorial. Evidently, the tutorial that helped students succeed on the two-source interference task did not help them transfer this understanding to a different context. It may be that the slit separation task is intrinsically more difficult than those in the context of circular wave fronts, perhaps because of the representation [24] used or the specific steps required to solve it. We reasoned that if this were the case, a 


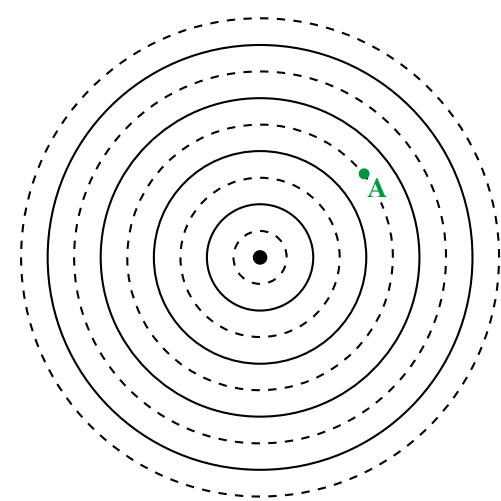

FIG. 5 (color online). Diagram shown to students as part of the single-source problem.

discrepancy in performance would emerge even before the Two-source interference tutorial. The results from a different question included in a modified pretest for the tutorial Two-source interference allow us to test this idea.

In the single-source problem, a set of circular wave fronts is indicated by concentric solid and dashed lines representing crests and troughs. (See Fig. 5.) A single source is described as "a small object tapping the water ... that moves up and down at a constant rate." A small piece of paper is described as floating on the surface of the water at a labeled point. Students are asked to determine the distance (in terms of $\lambda$ ) between the source and the piece of paper.

The answer to this question, $D=5 \lambda / 2$, can be determined by inspecting the diagram. About $77 \%$ of the students have answered this part correctly, significantly more than have answered the slit separation task correctly at the same stage. (See Table VI.) The results thus support the interpretation that the difference in performance is related to nature of the tasks. We note that on the slit separation task students must relate the distance between the slits to the distance between adjacent wave fronts, probably with the intermediate step of relating both to the square grid shown. Moreover, the two distances they must relate are mutually perpendicular. On the first part of the two-source interference task and on the single-source task, the relevant distances are marked out with lines representing crests and troughs and simple counting will suffice.

\section{Role of the context: Physics versus "everyday"}

The results we have presented thus far could still be explained in terms of students' framing of the task as one requiring something other than commonplace knowledge, because of the physicslike context and terminology of the problems (waves in ripple tanks) or simply the fact they are being asked the question as part of a physics course. While we have no data that address the second of these possibilities, we have asked students a similar question in a more "everyday" context. On the pencil task (see Fig. 6), students were asked to express the width $(\mathrm{AB})$ of a piece of construction paper in terms of the length of a pencil. On the basis of the reasoning required, we believe this task is analogous to the slit separation task. In both cases, the two distances are mutually perpendicular and can be related to one another by referencing the distances to the square grid in the background. We had two reasons for giving the pencil task: (1) to see if students would be more successful on a task involving a tool more similar to the familiar meter sticks and rulers used to measure distance, and (2) to see if the presence of such a task would spur more students to apply similar reasoning to distance tasks involving wavelengths as the unit of measure. (The second issue is discussed later.)

The pencil task has been given as part of the online pretest for the two-source interference tutorial. Between $85 \%$ and $95 \%$ of the students at NDSU and UW answered correctly, well above the percentage of correct answers on the slit separation task at the same stage of instruction. (See Table VI.) The percentage of correct answers on the pencil task is within the range of those obtained on the singlesource task (in fact it is within 1 standard deviation of the average); however, the single-source task is situated in a physics context. Thus there are no clear implications for the notion that presenting the task in a more physicslike context by itself has a negative influence on student performance. We note that the pencil and slit separation tasks differ in (at least) one other significant way: the pencil, being "concrete" and of fixed length, has more in common with the typical measurement devices most familiar to students (e.g., rulers, meter sticks) than with the changeable wavelength of a periodic wave. Thus, we think it is likely that the nature of the concept of wavelength is a

TABLE VI. Percentage of correct answers with correct reasoning for several tasks given at UW either before any instruction on two-source interference or after all instruction, including tutorial instruction. (Some of these data also appear in other tables; they are repeated here to facilitate comparisons.)

\begin{tabular}{lcc}
\hline \hline Timing relative to instruction (including tutorial instruction) & Before any & After all \\
\hline The slit separation problem & $35 \%$ & $58 \%$ \\
The single-source problem & $77 \%$ & not applicable \\
The pencil problem & $85 \%-95 \%$ & not applicable \\
Question A of the two-source interference problem & not applicable & $94 \%$ \\
(measuring the distance between two sources) & & \\
\hline \hline
\end{tabular}




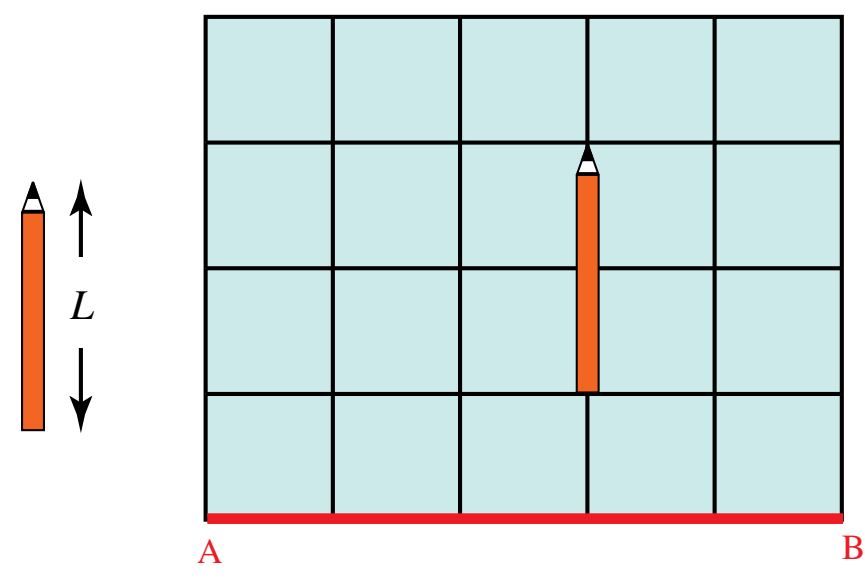

FIG. 6 (color online). Diagram shown to students as part of the pencil task.

significant factor in explaining student performance (e.g., students may think of wavelength as an abstract quantity that is not related to distance).

\section{Role of representation}

The failure to transfer basic skills for measuring distances in terms of nonstandard units to the context of the slit separation task may be attributed to student confusion about the nature of the task itself. If students do not understand the task or have a vague idea as to what the presented situation is all about, they may have difficulties recognizing what set of skills is applicable to this situation. We therefore hypothesized that interpreting the figure and placing it in the context of a real world situation may have been an issue for some students. We note that the difference in the success rate prior to any instruction and after all instruction (35\% and 58\% correct with correct reasoning, respectively, as shown in Table V) may reflect, in part, students' exposure to similar diagrams and a demonstration of periodic water waves moving through a slit.

We explored this possibility by designing a version of the online pretest in which students were first shown a video of waves generated by a dowel moving back and forth periodically in a shallow tank of water. Captions at the bottom of the screen described the setup and informed students that in the following pretest they "will be considering periodic water waves incident on a mask (barrier) with two narrow openings (slits)." (Neither a barrier nor an interference pattern was shown in order to avoid triggering students' expectations that the wave behavior on the other side of the slits determines the answer.) If confusion about this representation had been a significant issue on the slit separation task, we reasoned that students would be better able to interpret the figure associated with the task after viewing periodic plane water waves in the video, and the proportion of correct answers would increase.

The version with the video was given at UW and NDSU and the version without the video was given at UW only. The results summarized in Table VII suggest that placing the slit separation task (as well as the associated representations) in the context of a specific physics situation may help some students make sense of the task, as the percentage of correct student responses at UW did, in fact, increase by $\sim 18 \%$. However, the small gains in student performance on the original slit separation task due to the inclusion of the video are now approaching those made as a result of further instruction. This result supports our hypothesis that such gains may primarily stem from increased familiarity with the physics context and representation rather than from the transfer of skills acquired during instruction.

It is critical to note that student performance on the slit separation pretest with the video is still significantly worse than that on the directly analogous pencil task asked at the same point during instruction. Therefore, it seems unlikely that student confusion about the representation and physical context used in the slit separation task can account for the rather striking difference in student performance on these analogous tasks.

\section{Role of prompts to use commonsense reasoning}

As mentioned above, administering the pencil task served more than one purpose. In part we were interested in knowing whether the mere presence of this task would spur students to apply skills they demonstrate in some situations to those that are apparently more demanding. At both UW and NDSU, there was no apparent difference in student performance on subsequent parts of the pretest on which the pencil task appeared [25]. However, at NDSU, where the pencil task appeared on the pretest and the lecture instructor later invoked this task while discussing interference in lecture, performance on the two-source interference exam problem was significantly better than in previous semesters $(p<0.05)[26]$. At UW, on the other

TABLE VII. Percentage of correct answers with correct reasoning on the slit separation task before any instruction on two-source interference and either with or without a video intended to help students interpret the figure.

\begin{tabular}{lccc}
\hline \hline & UW & UW & NDSU \\
& $N=315$ & $N=195$ & $N=142$ \\
\hline Presence of a video intended to clarify the representation & Without & With & With \\
Correct with correct and complete reasoning & $35 \%$ & $53 \%$ & $42 \%$ \\
\hline \hline
\end{tabular}


TABLE VIII. Summary of results for parts D and E of the two-source interference problem after all instruction, and either with or without various prompts intended to help students use skills they had demonstrated in the context of concrete, everyday objects to the context of water waves.

\begin{tabular}{|c|c|c|c|c|c|}
\hline & \multicolumn{5}{|c|}{$\begin{array}{c}\text { Inclusion of pencil task } \\
\text { on pretest }\end{array}$} \\
\hline & \multicolumn{2}{|c|}{ Regular pretest } & \multirow{2}{*}{$\begin{array}{c}\text { With } \\
\text { solution } \\
\text { provided } \\
\\
\\
\mathrm{UW} \\
N=195\end{array}$} & \multirow{2}{*}{$\begin{array}{c}\text { Without } \\
\text { solution } \\
\text { provided } \\
\\
\text { UW } \\
N=152\end{array}$} & \multirow{2}{*}{$\begin{array}{c}\text { Inclusion of } \\
\text { pencil task on } \\
\text { pretest with } \\
\text { subsequent } \\
\text { discussion in } \\
\text { lecture } \\
\text { NDSU } \\
N=168\end{array}$} \\
\hline & $\begin{array}{c}\mathrm{UW} \\
N=153\end{array}$ & $\begin{array}{l}\text { NDSU } \\
N=87\end{array}$ & & & \\
\hline Correct answer with correct and complete reasoning on both questions & $22 \%$ & $6 \%$ & $13 \%$ & $11 \%$ & $21 \%$ \\
\hline $\begin{array}{l}\text { Correct answer on both questions with incomplete reasoning in D } \\
\text { " } d \text { in terms of } \lambda \text { increases, the \# of lines increases" }\end{array}$ & None & $14 \%$ & $4 \%$ & $1 \%$ & $13 \%$ \\
\hline \multicolumn{6}{|c|}{ Types of incorrect reasoning (on one or both questions) } \\
\hline Incorrect relationship among $v, f$ and $\lambda$ & $23 \%$ & $26 \%$ & $27 \%$ & $31 \%$ & $21 \%$ \\
\hline Difficulties with the concept of interference & $27 \%$ & $13 \%$ & $26 \%$ & $35 \%$ & $17 \%$ \\
\hline Incorrect $d$ or $\Delta D$ in terms of $\lambda$ & $9 \%$ & $8 \%$ & $13 \%$ & $15 \%$ & $10 \%$ \\
\hline
\end{tabular}

hand, where no additional reference was made to the pencil task, there was no positive shift in student performance on any parts of the two-source interference exam problem. (See Table VIII.) It may be that the students needed the additional prompt to see the connection between the two measurement schemas: one using a concrete object and the other using the regular spacing between wave fronts in a periodic wave. Our results suggest that the pencil task may have served as "preparation for future learning" [28]. The instructor's intervention built upon this task may have helped facilitate subsequent analogical transfer.

\section{CONCLUSION}

Many research publications and curriculum materials have focused on young children's experiences with the length measurement at the K-8 level. (See, for example, $[29,30]$.) However, we are not aware of any investigations that examine the ability of college students to express a distance in terms of nonstandard units (e.g., the wavelength of a periodic wave) in the context of physical optics. An ability to complete such a task correctly is so fundamental to understanding interference that we believe it warrants attention in instruction and research. In a series of different problems involving different contexts, and given at different stages of instruction, we found that many students failed to express a distance in terms of the wavelength of a periodic wave correctly. We have conducted a systematic investigation focused on the identification of factors influencing student performance on a variety of tasks that require measurements of distances in terms of nonstandard units.

On some tasks, as few as $50 \%$ (or less) of the students succeeded. The percentage of correct responses was not the only aspect that varied—some tasks elicited different types of responses when given at different stages in instruction. The more instruction students had, the more likely they were to treat the task as one requiring additional concepts related to wave motion or interference, leading some to claim there was insufficient information, and others to offer mathematical expressions that were both unnecessary and unhelpful. We considered the possibility that instruction on interference, and particularly instruction that emphasized mathematical analysis, was leading some students who would otherwise have answered correctly to frame the task inappropriately. We did not find support for this idea. In fact, the lack of significant change in the overall success rate as instruction progressed raised the possibility that further instruction was simply leading students who would otherwise have answered incorrectly to manipulate math equations in attempt to justify their incorrect ideas [31]. Nonetheless, it remains possible that the simple fact that students were given these tasks as part of a physics course was a contributing factor.

However, the types of incorrect responses we obtained suggest that for many students, the concept of wavelength itself is part of the problem. It was particularly striking that some students were able to apply the definition of $\lambda$ to mark a distance corresponding to a wavelength on a diagram, but were not able to use this definition in order to complete a simple task of expressing a specific distance in terms of $\lambda$. This finding suggested that these students either do not possess a functional understanding of wavelength or struggle with basic task of expressing distances in terms of nonstandard units. Student performance on the pencil task designed in a more everyday context does not support the latter possibility. Almost all students were able to express the width of the paper in terms of the 
pencil length, while many were not able to complete an analogous task in the context of the slit separation task. This suggests that perhaps students treat wavelength as an abstract quantity not related to distance. That, in turn, may hinder the transfer of basic measurement skills between two schemas: one using a concrete object and the other using the spacing between moving wave fronts of a periodic wave.

We note that instruction in a tutorial involving circular wave fronts on the water surface led to improvements, but they appear to be limited to the context in which they were learned. We are working on modifications to the sequence of tutorials in order to improve student ability to transfer. We have some evidence that instruction can help students make a connection between a task that they can do easily and one that is analogous but more cognitively demanding. Specifically, completion of the pencil task prior to attempting various wavelength tasks did not by itself lead to improvement on the latter, but when an instructor drew students' attention to this connection during a lecture, subsequent performance did improve. Thus, at least in this case, transfer did not happen automatically. Some formal instruction was necessary to help students recognize the applicability of the mastered skills to the new context.

Although the present discussion has primarily focused on issues of framing, transfer, and specific physics contexts, some responses hinted at the possibility of more general reasoning difficulties. For instance, a fraction of students seemed to be saying that two quantities that are not related to each other (e.g., distance between the slits is independent of a wave) cannot be expressed in terms of each other. It remains to be seen whether this tendency will arise in other contexts.
[1] K. Wosilait, P. R. L. Heron, P.S. Shaffer, and L. C. McDermott, Addressing student difficulties in applying a wave model to the interference and diffraction of light, Am. J. Phys. 67, S5 (1999).

[2] M. Kryjevskaia, M. R. Stetzer, and P. R. L. Heron, Student understanding of wave behavior at a boundary: The limiting case of reflection from fixed and free ends, Am. J. Phys. 79, 508 (2011).

[3] M. Kryjevskaia, M. R. Stetzer, and P. R. L. Heron, Student understanding of wave behavior at a boundary: The relationships among wavelength, propagation speed, and frequency, Am. J. Phys. 80, 339 (2012).

[4] L.C. McDermott, P.S. Shaffer, and the PEG at UW, Tutorials in Introductory Physics (Pearson/Prentice Hall, Upper Saddle River, NJ, 2002).

[5] T. O'Brien Pride, S. Vokos, and L. C. McDermott, The challenge of matching learning assessments to teaching goals: An example from the work-energy and impulsemomentum theorems, Am. J. Phys. 66, 147 (1998).

[6] S. Vokos, P.S. Shaffer, B.S. Ambrose, and L.C. McDermott, Student understanding of the wave nature of matter: Diffraction and interference of particles, Am. J. Phys. 68, S42 (2000).

[7] P. R. L. Heron, M.E. Loverude, P. S. Shaffer, and L. C. McDermott, Helping students develop an understanding of Archimedes' principle. Part II. Development of researchbased instructional materials, Am. J. Phys. 71, 1188 (2003).

[8] M. Kryjevskaia, A. Boudreaux, and D. Heins, "Assessing the flexibility of research-based instructional strategies: Implementing tutorials in introductory physics in the lecture environment" (unpublished).

[9] C. H. Crouch and E. Mazur, Peer instruction: Ten years of experience and results, Am. J. Phys. 69, 970 (2001).

[10] Half of the students in the large enrollment course at NDSU were given the two-source interference question on the final course exam, while the other half were given the slit separation question.

[11] A quarter of the students wrote the correct equation, but failed to apply it correctly. Most of the students incorrectly predicted that the value of $\Delta D$ for the first nodal line must be either $3 \lambda / 2$ or $\lambda$.

[12] We believe such responses are related to the algebra "reversal error." This error tends to arise when students are told, for example, that "there are two professors for every 100 students," and then express this mathematically as $2 \mathrm{P}=100 \mathrm{~S}$, implying a professor-student ratio of 50:1, rather than 1:50. See, for example, [13]. For a discussion in the context of physics instruction, see [14].

[13] J. Clement, J. Lochhead, and G. S. Monk, Translation difficulties in learning mathematics, Am. Math. Mon. 88, 286 (1981).

[14] E. Cohen and S. E. Kanim, Factors influencing the algebra "reversal error," Am. J. Phys. 73, 1072 (2005).

[15] It is necessary for the students to be able to answer this question correctly in order to determine whether, after the change, an observer would see a complete constructive interference or a dark spot.

[16] E. M. Kennedy and J.R. de Bruyn, Understanding of mechanical waves among second-year physics majors, Can. J. Phys. 89, 1155 (2011).

[17] P. R. L. Heron, Student performance on conceptual questions: Does instruction matter?, AIP Conf. Proc. 1513, 174 (2013).

[18] D. Maloney, An overview of physics education research on problem solving, Getting Started in PER: Reviews in PER 2, http://www.per-central.org/per_reviews/ volume2.cfm\#V2I2.

[19] J. H. Larkin, J. McDermott, D. P. Simon, and H. A. Simon, Expert and novice performance in solving physics problems, Science 208, 1335 (1980). 
[20] D. Hammer, A. Elby, R.E. Scherr, and E.F. Redish, Resources, framing, and transfer, in Transfer of Learning: Research and Perspectives, edited by J. Mestre (Information Age Publishing, Greenwich, CT, 2004).

[21] Because of a scheduling conflict, it was not possible to give this task at NDSU.

[22] We note that the version of the slit separation problem given most often does not explicitly state that the figure is drawn to scale, which could account for some students' assertion that not enough information is supplied. However, when we have added a note to this effect there has been no noticeable difference in the results.

[23] A different version of the pretest was given at NDSU that contained a video of periodic plane waves generated by a dowel. The video pretest is discussed later in the paper.

[24] P. Kohl and N. Finkelstein, Effects of representation on students solving physics problems: A fine-grained characterization, Phys. Rev. ST Phys. Educ. Res. 2, 010106 (2006).
[25] These tasks are not discussed in this paper but are discussed in the paper cited in Ref. [1].

[26] We compared the percentages of correct responses on parts $\mathrm{D}$ and $\mathrm{E}$ after the regular pretest and after the pretest with the inclusion of the pencil task followed by a discussion in lecture (see Table VIII). The two-tailed $p$ value is calculated based on the two-proportion $z$-test. See, for example, Ref. [27].

[27] F. L. Ramsey and D. W. Schafer, The Statistical Sleuth: A Course in Methods of Data Analysis (Cengage Learning, Inc., Belmont, CA, 1996), 1st ed., Chap. 18.

[28] J. D. Bransford and D. L. Schwartz, Rethinking transfer: A simple proposal with multiple implications, Rev. Res. Educ. 24, 61 (1999).

[29] D. Clements, Teaching length measurement: Research challenges, School Sci. Math. 99, 5 (1999).

[30] A. MacDonald and T. Lowrie, Developing measurement concepts within context: Children's representations of length, Math. Educ. Res. J. 23, 27 (2011).

[31] M. Kryjevskaia and M. R. Stetzer, Examining inconsistencies in student reasoning approaches, AIP Conf. Proc. 1513, 226 (2013). 